\title{
The impact of sleep quality and duration on leptin, appetite, and obesity indices in adults
}

Harry Freitag Luglio Muhammad, ${ }^{1}$ Satwika Arya Pratama, ${ }^{1}$ Dian Caturini Sulistyoningrum, ${ }^{1}$ Maya Nurfitriani Hartono, ${ }^{1}$ Emy Huriyati, ${ }^{1}$ Yi Yi Lee, ${ }^{2}$ Wan Abdul Manan Wan Muda ${ }^{3}$

Check for updates

pISSN: 0853-1773 - elSSN: 2252-8083 https://doi.org/10.13181/mji.oa.203707 Med J Indones. 2020;29:417-21

Received: April 11, 2019

Accepted: August 14, 2020

Authors' affiliations:

${ }^{1}$ Faculty of Medicine, Public Health and Nursing, Universitas Gadjah Mada, Yogyakarta, Indonesia, ${ }^{2}$ Division of Nutrition and Dietetics, International Medical University, Kuala Lumpur, Malaysia, ${ }^{3}$ Department of Nutrition Science, Faculty of Health Science, Universitas Alma Ata, Yogyakarta, Indonesia

\section{Corresponding author:}

Harry Freitag Luglio Muhammad Faculty of Medicine, Public Health and Nursing, Universitas Gadjah Mada, Jalan Farmako Sekip Utara, Yogyakarta 55281, Indonesia

Tel/Fax: +62-274-547775

E-mail: harryfreitag@ugm.ac.id

\begin{abstract}
BACKGROUND Poor sleep duration and quality were previously reported to increase the risk of obesity. This study was aimed to evaluate the impact of sleep quality and duration on leptin, appetite, and adiposity in Indonesian adults.
\end{abstract}

METHODS This cross-sectional study recruited adults in Yogyakarta, Indonesia, in 2016. Sleep quality was assessed using the Pittsburgh sleep quality index, appetite was evaluated using the community nutrition appetite questionnaire, and dietary intake was assessed by interviews using the semi-quantitative food frequency questionnaire. Sleep duration was also asked. Obesity indices were measured using the body mass index (BMI), waist-hip circumference, and percentage of body fat. Leptin was analyzed using the enzyme-linked immunosorbent assay. A Spearman analysis was done to evaluate the correlation between sleep quality, sleep duration, anthropometric measures, dietary intake, appetite, and leptin.

RESULTS 244 adults participated in this study. This study showed that lower sleep quality and duration was significantly correlated with higher body weight $(r=0.129$, $p=0.043$ and $r=-0.228, p<0.001)$, BMI $(r=0.176, p=0.006$ and $r=-0.202, p=0.001)$, and waist circumference $(r=0.179, p=0.005$ and $r=-0.254, p<0.001)$. There was a correlation between poor sleep quality and higher leptin concentration $(r=0.186, p$ $=0.004)$. Sleep quality and duration were not associated with appetite $(r=0.109, p=$ 0.109 and $r=-0.043, p=0.500$ ).

CONCLUSIONS This study found that lower sleep quality was correlated with higher $\mathrm{BMI}$, higher leptin concentration, but not appetite.

KEYWORDS appetite, leptin, obesity, sleep quality, sleep duration
Good quality sleep and sufficient sleep duration are essential components for human health. Those who habitually sleep outside the normal range might have consequential effects on their health and well-being. ${ }^{1}$ Poor sleep is often associated with an increased risk for injuries (traffic- or occupation-related), reduced productivity, impaired mood, higher body mass, and development of noncommunicable diseases (NCDs). ${ }^{2}$ It has been suggested that there has been an increasing problem with sleep duration and quality in recent decades, especially among adult individuals who live in urban areas. This disturbance in sleep duration and quality has been observed in several countries, such as the United States, Europe, and Asia. ${ }^{2-4}$

Disturbances in sleep quality and quantity were associated with changes in human body homeostasis. These include changes in the hypothalamus-pituitaryadrenal system, sympathetic nervous system, insulin sensitivity, leptin, and ghrelin. ${ }^{5,6}$ These changes will induce reductions in energy expenditure and increases 
in appetite, which, in turn, increase the risk for obesity and the development of NCDs. ${ }^{6-8}$ Investigating the role of sleep quality and duration of obesity is essential because obesity is responsible for an increasing economic and health burden worldwide.9,10 This study was aimed to evaluate the impact of sleep quality and duration on leptin, appetite, and obesity indices in Indonesian adults.

\section{METHODS}

This investigation was a cross-sectional study conducted among adult males and females in the urban area of Yogyakarta, Indonesia. The inclusion criteria were age between 18 and 56 years old, permanent resident of the area for at least 2 years, and agreement to become a subject of this study. Those diagnosed with having degenerative diseases by a medical doctor, were pregnant, and breastfeeding were excluded from this study. Subjects with a strict, prescribed diet or a problem performing physical activity in the last 6 months were also excluded from this study. This study has been approved by the Medical and Health Research Ethics Committee Faculty of Medicine, Universitas Gadjah Mada, Indonesia (KE/FK/791/EC/2015). All subjects provided informed consent before data collection.

Obesity indices included body mass index (BMI), waist-hip circumference, and body fat percentage. $\mathrm{BMI}$ was determined by dividing body weight $(\mathrm{kg})$ by height $(\mathrm{m})$ squared $\left(\mathrm{m}^{2}\right)$. Body weight and percentage of body fat were measured using a digital body mass scale (Omron, Japan) with an accuracy of $0.1 \mathrm{~kg}$. Height was measured using a wall-mounted tape measure (GEA Medical, Indonesia) with an accuracy of $0.1 \mathrm{~cm}$. A non-elastic tape was used to measure the waist and hip circumferences with an accuracy of $0.1 \mathrm{~cm}$.

Dietary intake, appetite, sleep quality, and sleep duration were measured by direct interviews. Dietary intake and food consumption were assessed using a semiquantitative food frequency questionnaire. The analysis was performed using the composition of nutrients from the Indonesian food database and the United States Department of Agriculture by the researchers (HFLM). The dietary intake was reported as the gram intake corrected for the total energy intake, gram/1,000 kcal. This was calculated by dividing grams of nutrient intake with total energy intake, and the result was multiplied by 1,000 kcal.
The sleep quality of subjects was determined by the Pittsburgh sleep quality index (PSQI). ${ }^{11}$ The PSQI was chosen because of its reliability for evaluating sleep quality in a population. ${ }^{12} \mathrm{An}$ increase in the PSQI score shows a reduction in sleep quality. Sleep quality was determined as poor if the PSQI score $>5$ points. Sleep duration was the total hours spent sleeping in a previous 24-hour period.

Appetite was measured using the community nutrition appetite questionnaire. ${ }^{13}$ This questionnaire was developed to assess the appetite of individuals in a community setting. This questionnaire was initially designed for older adults but has been validated for younger individuals.

The measurement of leptin plasma was done under nonfasting conditions (DRG International Inc., USA). Ten $\mathrm{ml}$ of blood was collected in ethylenediaminetetraacetic acid-containing tubes. Within 2 hours after collection, the blood plasma and buffy coat were separated using a centrifuge. The plasma leptin was measured using an enzyme-linked immunosorbent assay (DRG International Inc.).

JASP software 0.8.3.1 was used for the statistical analysis (The University of Amsterdam, The Netherlands). Subjects were divided into two groups: those with poor sleep quality and those with good sleep quality. The difference in leptin, appetite, dietary intake, and obesity indices between good and poor sleep quality was analyzed using an independent $t$-test or Mann-Whitney test. The correlation between sleep quality, sleep duration, anthropometric measures, dietary, appetite, and leptin was evaluated by performing a Spearman analysis.

\section{RESULTS}

There were 244 adults who participated in this study. Their characteristics are shown in Table 1. The average BMI of the subjects was $25.1 \mathrm{~kg} / \mathrm{m}^{2}$ (14.5$46.4 \mathrm{~kg} / \mathrm{m}^{2}$ ). In this study, $18.4 \%$ were obese, $25 \%$ were overweight, $50.4 \%$ were normal weight, and $6.1 \%$ were underweight. There were $59.8 \%$ subjects had poor sleep quality and $61.1 \%$ subjects had sleep duration $<7$ hours a day. Meanwhile, there were $47.9 \%$ who had both poor sleep quality and sleep duration $<7$ hours a day.

Subjects were equally distributed between males (47.5\%) and females (52.4\%). In comparison to females, males had a higher body weight $(p=0.020)$ 
and height $(p<0.001)$, but lower BMI $(p<0.001)$, waist circumference $(p=0.001)$, hip circumference $(p<0.001)$ and body fat $(p<0.001)$. In addition, male subjects had better sleep quality $(p=0.032)$ but not sleep duration $(p=0.509)$.

The association between sleep quality, anthropometric measures, and leptin is presented in Table 1. Sleep quality was determined based on PSQI scores. Males were associated with better sleep quality than females $(p=0.028)$. We reported that poor sleep quality was related to higher BMI $(p=0.011)$, waist circumference $(p=0.008)$, hip circumference $(p=0.023)$, and body fat $(p=0.026)$.
In addition, poor sleep quality was associated with higher leptin concentration $(p=0.004)$. Appetite was not associated with sleep quality $(p=0.430)$. We also reported that dietary intake and typical food consumption were not associated with sleep quality (all $p>0.05$ ).

Correlation between sleep quality, sleep duration, anthropometric measures, leptin, appetite, and dietary intake were shown in Table 2. In this study, we showed that all anthropometric measures and leptin were positively correlated with the PSQI score. The correlation between sleep quality and appetite was not correlated.
Table 1. Characteristics of subjects based on sleep quality
Table 2. Correlation between sleep, anthropometric measures, leptin, appetite, and dietary intake

\begin{tabular}{|c|c|c|c|c|}
\hline & $\begin{array}{l}\text { All subjects } \\
(\mathrm{N}=244)\end{array}$ & $\begin{array}{l}\text { Poor quality* } \\
\qquad(N=146)\end{array}$ & $\begin{array}{l}\text { Good quality } \\
\qquad(N=98)\end{array}$ & $p$ \\
\hline Age $(\text { years) })^{\ddagger}$ & $41.4(0.6)$ & $42.0(0.9)$ & $40.6(0.9)$ & 0.276 \\
\hline Sex $(\text { male } / \text { female })^{\S}$ & $116 / 128$ & $61 / 85$ & $55 / 43$ & 0.028 \\
\hline \multicolumn{5}{|l|}{ Anthropometric measures $^{\ddagger}$} \\
\hline Body weight (kg) & $62.6(0.9)$ & $63.8(1.2)$ & $60.8(1.3)$ & 0.081 \\
\hline Height $(\mathrm{cm})$ & $158.2(0.6)$ & $157.5(0.8)$ & $159.1(0.9)$ & 0.196 \\
\hline $\mathrm{BMI}\left(\mathrm{kg} / \mathrm{m}^{2}\right)$ & $25.1(0.4)$ & $25.8(0.5)$ & $24.0(0.5)$ & 0.011 \\
\hline Waist circumference $(\mathrm{cm})$ & $87.0(0.9)$ & $88.8(1.2)$ & $84.2(1.2)$ & 0.008 \\
\hline Hip circumference $(\mathrm{cm})$ & $94.2(0.8)$ & $95.6(1.1)$ & $92.0(1.1)$ & 0.023 \\
\hline Body fat (\%) & $27.8(0.6)$ & $28.9(0.8)$ & $26.1(0.9)$ & 0.026 \\
\hline Leptin $(\mathrm{ng} / \mathrm{ml})^{\ddagger}$ & $7.8(0.6)$ & $9.3(0.9)$ & $5.7(0.8)$ & 0.004 \\
\hline
\end{tabular}

$\mathrm{SE}=$ standard error; $\mathrm{BMI}=$ body mass index; PSQI=Pittsburgh sleep quality index *Independent $t$-test; ${ }^{\dagger}$ based on PSQI; ${ }^{\sharp}$ Mann-Whitney test; ${ }^{\S}$ chi-square test

\begin{tabular}{lcccc}
\hline & \multicolumn{2}{c}{ Sleep quality (PSQI score) } & \multicolumn{2}{c}{ Sleep duration (hours) } \\
\cline { 2 - 5 } & $\mathrm{r}^{*}$ & $p$ & $\mathrm{r}^{*}$ & $p$ \\
\hline Weight $(\mathrm{kg})$ & 0.129 & $\mathbf{0 . 0 4 3}$ & -0.228 & $<\mathbf{0 . 0 0 1}$ \\
\hline BMI (kg/m²) & 0.176 & $\mathbf{0 . 0 0 6}$ & -0.202 & $\mathbf{0 . 0 0 1}$ \\
\hline Waist circumference (cm) & 0.179 & $\mathbf{0 . 0 0 5}$ & -0.254 & $<\mathbf{0 . 0 0 1}$ \\
\hline Hip circumference (cm) & 0.185 & $\mathbf{0 . 0 0 4}$ & -0.191 & $\mathbf{0 . 0 0 3}$ \\
\hline Body fat (\%) & 0.172 & $\mathbf{0 . 0 0 7}$ & -0.103 & 0.110 \\
\hline Leptin (ng/ml) & 0.186 & $\mathbf{0 . 0 0 4}$ & -0.095 & 0.141 \\
\hline Appetite rating (CNAQ score) & 0.103 & 0.109 & -0.043 & 0.500 \\
\hline Dietary intake (daily) & & & & \\
\hline Energy (kcal) & -0.052 & 0.416 & 0.067 & 0.295 \\
\hline Protein (g/1,000 kcal) & -0.076 & 0.239 & 0.080 & 0.211 \\
\hline Fat (g/1,000 kcal) & -0.045 & 0.489 & 0.034 & 0.594 \\
\hline Carbohydrate (g/1,000 kcal) & 0.075 & 0.244 & -0.050 & 0.437 \\
\hline Fiber (g/1,000 kcal) & 0.003 & 0.962 & 0.084 & 0.191 \\
\hline Sugar (g/1,000 kcal) & 0.038 & 0.551 & -0.032 & 0.622 \\
\hline
\end{tabular}

PSQI=Pittsburgh sleep quality index; $\mathrm{BMI}=$ body mass index; $\mathrm{CNAQ=community} \mathrm{nutrition} \mathrm{appetite}$ questionnaire

*Spearman correlation test 


\section{DISCUSSION}

We reported that poor sleep quality was associated with higher leptin and all obesity indices, but not appetite. In addition, this study showed a negative correlation between sleep duration and obesity indices. Our study indicated that sleep patterns might play a role in the development of obesity. Thus, achieving good sleep quality and sufficient sleep duration might be important lifestyle components to consider for obesity prevention.

Adults with poor sleep quality and duration were associated with higher adiposity indices. Findings from this study were similar to the findings of several studies that showed a correlation between sleep duration and body composition. ${ }^{5}$ It was previously reported that longer sleep duration was associated with lower BMI and body fat. ${ }^{5}$ This association was also reported in children and adolescents. ${ }^{5}$

Several mechanisms have been investigated regarding the impact of sleep disturbances on the regulation of body weight. These mechanisms include altered metabolic parameters, appetite-related hormones, the sympathetic nervous system's activity, appetite, and energy expenditure. ${ }^{5}$ Subjects with lower sleep duration, especially reduced rapid eye movement sleep and slow-wave sleep, were associated with a reduction in energy balance, leading to a higher chance of energy storage. In addition, shorter sleep duration has been associated with a reduction of leptin (satiety inducing hormone) and an incremental increase in ghrelin (hunger-inducing hormone), leading to an increase in appetite. ${ }^{5}$

Sleep deprivation has been associated with a disruption in the activity of the appetitive evaluation region in the human brain. This condition, in addition to the up-regulation of orexin neurons and changes in appetite-regulating hormones, ${ }^{14}$ is followed by the increasing desire to eat, especially high-calorie foods. ${ }^{15}$ In this study, we showed that, sleep quality was not associated with appetite and dietary intake. It is argued that appetite is not the single driver of individual dietary intake. Some other factors might also play a role such as socioeconomic factors, age, and food availability.

There has been conflicting evidence regarding the association between sleep and plasma leptin concentration. In a cross-sectional study of individuals with obese and type 2 diabetes mellitus, Hirota et al ${ }^{16}$ reported that sleep quality was positively associated with leptin concentration. By contrast, Knutson et al ${ }^{17}$ reported that in obese males and females, leptin was not associated with sleep quality and duration. Several studies that supported our findings demonstrated that poor sleep quality was associated with higher leptin concentration. ${ }^{18-21}$ Van Leeuwen et $\mathrm{al}^{18}$ showed that prolonged sleep restriction was correlated with increased leptin concentration. Investigation in 443 police officers showed that sleep duration was associated with leptin concentration. ${ }^{19}$ This effect was seen in males and females. ${ }^{20,21}$ Leptin has an essential role in increased systemic inflammation. This also suggests that the increasing concentration of leptin because of poor sleep quality would potentially increase the risk for metabolic syndrome and cardiovascular diseases in the future. ${ }^{22}$

In contrast to sleep quality, the relationship between sleep duration and leptin is unclear. A systemic review and meta-analysis recently reported the association between sleep restriction, leptin, obesity, hunger, and energy intake. ${ }^{23}$ It stated that sleep restriction was associated with weight gain, increased energy intake, and hunger. However, the meta-analysis concluded that there was no association between sleep restriction and leptin. It is argued that some factors, including circadian rhythm and the inert nature of leptin release, might influence its response to sleep restriction. ${ }^{23}$ It is still unclear how sleep quality and duration could affect leptin concentration. It has been suspected that shorter sleep duration could increase sympathetic activity and stress signals, which, in turn, could increase leptin concentration. ${ }^{24}$ The authors also suggested that this might be due to the reduction in leptin sensitivity because of poor sleep quality.

There were several limitations to this study. Subjects were in their free-living condition; thus, an objective sleep analysis cannot be used in this study. Instead, a subjective assessment of sleep quality and duration was used because of its reliability in a community setting. ${ }^{15} \mathrm{~A}$ causal-effect of both variables cannot be drawn in this study because of its cross-sectional design. Leptin was measured in a nonfasting condition because of the practicality for data collection in a community setting. This might influence the variability of leptin levels between subjects. To our knowledge, limited studies are investigating the relationship between sleep parameters and leptin in a free-living populationbased study. Further studies are needed to evaluate 
whether sleep improvement strategies could be used as an alternative to obesity prevention.

In conclusion, we reported that poor sleep quality and duration were associated with increased obesity indices among adults. Lower sleep quality also correlated with higher leptin. Lifestyle recommendations for obesity prevention should emphasize the importance of good sleep quality and sufficient sleep duration.

\section{Conflict of Interest}

The authors affirm no conflict of interest in this study.

\section{Acknowledgment}

We thank Mohammad Zaenal Sofro for his advice. His advice helped improve the design of this study. We also thank Cita Eri Ayuningtyas for her help during data collection.

\section{Funding Sources}

This study was funded by International Research Collaboration (IRec) Grant (IREC Grant No: 1002/PPSK/910408) (Universiti Sains Malaysia), Community Fund-Faculty of Medicine (Universitas Gadjah Mada), and Young Investigator Awards (Universitas Gadjah Mada).

\section{REFERENCES}

1. Hirshkowitz M, Whiton K, Albert SM, Alessi C, Bruni O, DonCarlos $\mathrm{L}$, et al. National Sleep Foundation's sleep time duration recommendations: methodology and results summary. Sleep Health. 2015;1(1):40-3.

2. Hoyos C, Glozier N, Marshall NS. Recent evidence on worldwide trends on sleep duration. Curr Sleep Med Rep. 2015;1:195-204.

3. Ryu JY, Lee JS, Hong HC, Choi HY, Yoo HJ, Seo JA, et al. Association between body size phenotype and sleep duration: Korean National Health and Nutrition Examination Survey V (KNHANES V). Metab Clin Exp. 2015;64(3):460-6.

4. Sun W, Huang Y, Wang Z, Yu Y, Lau A, Ali G, et al. Sleep duration associated with body mass index among Chinese adults. Sleep Med. 2015;16(5):612-6.

5. Gonnissen HK, Adam TC, Hursel R, Rutters F, Verhoef SP, Westerterp-Plantenga MS. Sleep duration, sleep quality and body weight: parallel developments. Physiol Behav. 2013;121:1126.

6. McNeil J, Doucet E, Chaput JP. Inadequate sleep as a contributor to obesity and type 2 diabetes. Can J Diabetes. 2013;37(2):103-8.

7. Golem DL, Martin-Biggers JT, Koenings MM, Davis KF, ByrdBredbenner C. An integrative review of sleep for nutrition professionals. Adv Nutr. 2014;5(6):742-59.

8. Rahe C, Czira ME, Teismann H, Berger K. Associations between poor sleep quality and different measures of obesity. Sleep
Med. 2015;16(10):1225-8.

9. GBD 2015 Obesity Collaborators, Afshin A, Forouzanfar MH, Reitsma MB, Sur P, Estep K, et al. Health effects of overweight and obesity in 195 countries over 25 years. N Engl J Med. 2017;377(1):13-27.

10. Tremmel M, Gerdtham UG, Nilsson PM, Saha S. Economic burden of obesity: a systematic literature review. Int J Environ Res Public Health. 2017;14(4):435.

11. Buysse DJ, Reynolds CF, Monk TH, Berman SR, Kupfer DJ. The Pittsburgh Sleep Quality Index (PSQI): a new instrument for psychiatric research and practice. Psychiatry Res. 1989;28(2):193-213.

12. Hinz A, Glaesmer H, Brähler E, Löffler M, Engel C, Enzenbach $C$, et al. Sleep quality in the general population: psychometric properties of the Pittsburgh Sleep Quality Index, derived from a German community sample of 9284 people. Sleep Med. 2017;30:57-63.

13. Wilson MM, Thomas DR, Rubenstein LZ, Chibnall JT, Anderson S, Baxi A, et al. Appetite assessment: simple appetite questionnaire predicts weight loss in community-dwelling adults and nursing home residents. Am J Clin Nutr. 2005;82(5):1074-81.

14. Beccuti G, Pannain S. Sleep and obesity. Curr Opin Clin Nutr Metab Care. 2011;14(4):402-12.

15. Greer SM, Goldstein AN, Walker MP. The impact of sleep deprivation on food desire in the human brain. Nat Commun. 2013;4:2259.

16. Hirota T, Morioka T, Yoda K, Toi N, Hayashi N, Maruo S, et al. Positive association of plasma leptin with sleep quality in obese type 2 diabetic patients. J Diabetes Investig. 2018;9(5):1100-5.

17. Knutson KL, Galli G, Zhao X, Mattingly M, Cizza G, NIDDK Sleep Extension Study. No association between leptin levels and sleep duration or quality in obese adults. Obesity (Silver Spring). 2011;19(12):2433-5.

18. Van Leeuwen WMA, Hublin C, Sallinen $M$, Härmä $M$, Hirvonen A, Porkka-Heiskanen T. Prolonged sleep restriction affects glucose metabolism in healthy young men. Int J Endocrinol. 2010;2010:108641.

19. Charles LE, Gu JK, Andrew ME, Violanti JM, Fekedulegn D, Burchfiel CM. Sleep duration and biomarkers of metabolic function among police officers. J Occup Environ Med. 2011;53(8):831-7.

20. Reynolds AC, Dorrian J, Liu PY, Van Dongen HP, Wittert GA, Harmer LJ, et al. Impact of five nights of sleep restriction on glucose metabolism, leptin and testosterone in young adult men. PLoS One. 2012;7(7):e41218.

21. Simpson NS, Banks S, Dinges DF. Sleep restriction is associated with increased morning plasma leptin concentrations, especially in women. Biol Res Nurs. 2010;12(1):47-53.

22. Hou N, Luo JD. Leptin and cardiovascular diseases. Clin Exp Pharmacol Physiol. 2011;38(12):905-13.

23. Zhu B, Shi C, Park CG, Zhao X, Reutrakul S. Effects of sleep restriction on metabolism-related parameters in healthy adults: a comprehensive review and meta-analysis of randomized controlled trials. Sleep Med Rev. 2019;45:18-30.

24. Pan W, Kastin AJ. Leptin: a biomarker for sleep disorders? Sleep Med Rev. 2014;18(3):283-90. 